\title{
DiCa: Distributed Tag Access with Collision-Avoidance Among Mobile RFID Readers
}

\author{
Kwang-il Hwang, Kyung-tae Kim, and Doo-seop Eom \\ Department of Electronics and Computer Engineering, Korea University \\ 5-1ga, Anam-dong, Sungbuk-gu, Seoul, Korea \\ Tel: +82-2-3290-3802, Fax: +82-2-3290-3895 \\ brightday@final.korea.ac.kr
}

\begin{abstract}
Advances in wireless and mobile communication technologies have enabled the development of various RFID-based systems and applications in addition to the extension of the tag reading range of mobile readers. Thus, it has become commonplace that multiple readers concurrently attempt to read tags within ranges of the readers. However, this concurrent access among multiple mobile readers brings about a new problem called reader collision, where a reader's transmission is interfered by other readers. There have been several studies focusing on solving the reader collision problem. These studies employ time division, frequency division, space division, or the centralized scheduling approach. In this paper, a cooperative, distributed reader collision avoidance algorithm is introduced. In particular, the proposed DiCa (Distributed Tag Access with Collision-Avoidance) is considerably suitable for energy-efficient wireless mobile network environments cooperated with RFID, since the DiCa is capable not only of avoiding collisions, but also changing power states autonomously through simple interaction of adjacent readers.
\end{abstract}

\section{Introduction}

Automatic identification (Auto-ID) systems have become commonplace in industries requiring the identification of products at the point of sale or point of service as well as in access control and security applications [1]. Radio Frequency Identification (RFID) is an Auto-ID technique which uses a specific radio frequency to automatically identify data stored on a tag, label or card with a microchip, and tracks each item through a supply chain. A RFID system consists of a RFID reader, and tags, which are generally attached to an object. As the development of foundation technology of RFID progresses rapidly, readers that read information from tags are becoming smaller, cheaper, and more portable. This has enabled the development of the mobile RFID reader, beyond standalone readers. In particular, in recent, it is increased that mobile RFID readers are used in a traditional wireless network. In particular, sensor networks, which construct wireless multi-hop networks with innumerable small devices, due to 
the low cost, small, and distributed characteristics [2], is expected as one of the most suitable network technologies for interacting with RFID.

In such a mobile environment, it is commonplace for multiple readers to attempt to concurrently read a tag existing within ranges of both readers. However, this concurrent access among multiple mobile readers brings a new problem called reader collision, where a reader's transmission interferes with other readers. This reader collision problem [3] is considerably different from tag collision, which was considered a critical issue in traditional RFID systems. In recent years, there have been several studies focusing on solving the reader collision problem. While these studies employ time division, frequency division, space division, or centralized scheduling approaches, in this paper, a cooperative, distributed reader collision avoidance algorithm is introduced. In particular, the proposed DiCa (Distributed Tag Access with Collision-Avoidance) is considerably suitable for energy-efficient wireless mobile network environments cooperated with RFID, since the proposed DiCa (Distributed Tag Access with Collision-Avoidance) is capable of not only avoiding collisions, but also changing power state autonomously through simple interaction between adjacent readers.

The remainder of this paper is organized as follows. In section 2, characteristics of mobile readers are described. In section 3, work relating to the reader collision problem is presented. The proposed DiCa is introduced in Section 4. Performance of $\mathrm{DiCa}$ is evaluated in terms of throughput and energy efficiency in section 5 . Finally, this paper is concluded in section 6.

\section{Characteristics of Mobile Readers}

In contrast to stationary RFID readers, mobile RFID readers are available to freely read tags by moving the reader's position. The mobility of the RFID reader has several advantages as follows: coverage extension of readers, facilitation of inventory or stock, and low cost of installment and maintenance.

These advantages accelerated the use of mobile readers in various environments. However, mobile readers based on mobility can bring about serious problems of interference among multiple readers within a similar range. Fig. 1 illustrates interference which can be caused when two readers attempt to concurrently read a tag within an overlapping range. A collision by such interference is called reader collision. This reader collision must be avoided because collisions do not occur at the reader, but at the tag. Therefore readers cannot realize the fact that, due to collision, tag data is missed. The more the $\mathrm{RF}$ radio range and numbers of reader's increases, the more collisions occur. Therefore, reader collision should be avoided, as well as anti-collision among tags.

\section{Related Work}

In this section, work relating to the RFID reader collision problem is illustrated, and several limitations and problems of traditional algorithms are discussed. The first representative algorithm is Colorwave [5] which is a distributed TDMA 


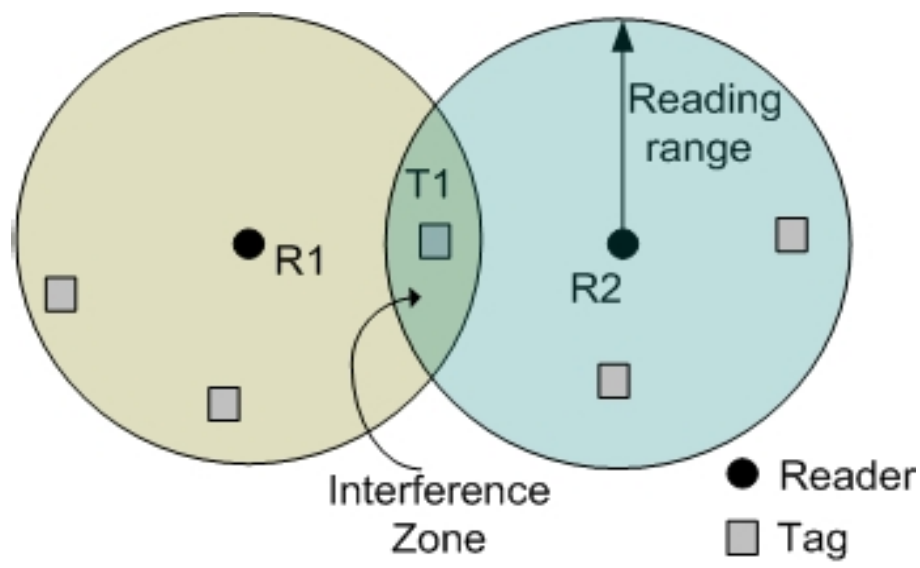

Fig. 1. Interference between readers

based algorithm. Each reader of a local RFID network chooses one of the slotted colors in [0, Maxcolors] randomly, and only a reader with a queued request for transmission can transmit data in its color timeslot. If the transmission collides with another reader, the transmission request is discarded, and the reader randomly chooses a new color and reserves this color. If a neighbor has the same color, it chooses a new color and transmits a kick packet. Each reader synchronizes with other readers by continuously tracking the current time slot. The number of Maxcolors varies according to the network situation. Colorwave enables the RFID system to easily adapt to local disturbances, based on local information, such as the installation of a new reader or the presence of a mobile RFID reader. However, Colorwave requires tight time synchronization between readers. Therefore, in Colorwave, overhead of time-slot reselection continuously increases when network topology is changed by reader mobility. Furthermore, Colorwave assumes that a reader is able to detect collisions in the network without being aware of a tag. However, the assumption is not suitable for feasible RFID systems.

Q-learning [6] presents online learning algorithms in hierarchical network architecture. In this algorithm, reader collision problem can be solved by learning collision patterns of readers and by effectively assigning frequencies to the readers over time. HiQ algorithm in Q-learning is composed of three basic hierarchical layers: reader, reader-level server, and Q-learning server. The readers transmit collision information to the upper layer server. An individual upper layer server then assigns resources to its readers. However, the hierarchical architecture requires additional managements in overall hierarchy despite a slight change in the lower layer. Therefore, in highly mobile environments, management overhead exponentially increases and, eventually, serious collisions or delay in the network is created.

Pulse [4 is similar to the proposed $\mathrm{DiCa}$ in that the algorithm attempts to solve the reader collision problem using two separated channels in the RFID 
system. One channel (data channel) is used for reader-tag communication and another (control channel) is used for reader-reader communication. That is, transmission on a control channel will not affect on-going communications on the data channel. The pulse algorithm presents a notification mechanism where a reader transmits a broadcast message called "beacon" to its neighbors periodically through the control channel, while it is communicating with the tags through the data channel. Although the Pulse is similar to the presented DiCa in that reader collision is avoided by the two independent channels, the Pulse has inherent overhead for broadcasting periodic beacon in all nodes. In addition, the hidden and exposed problems from using two channels are still unsolved. In contrast to previous studies, the proposed DiCa does not require any centralized coordinator or global synchronization. In addition, the DiCa copes well with the hidden and exposed problems by employing variable power control.

\section{DiCa: Distributed Tag Access with Collision Avoidance}

In this section, a cooperative, distributed reader collision avoidance algorithm is presented. The main design goal of the proposed DiCa (Distributed Tag Access with Collision-Avoidance) is energy-efficiency and compatibility with other access networks, as well as collision avoidance. In particular, the DiCa is distinguished from other reader collision avoidance methods due to its distributed collision avoidance characteristics.

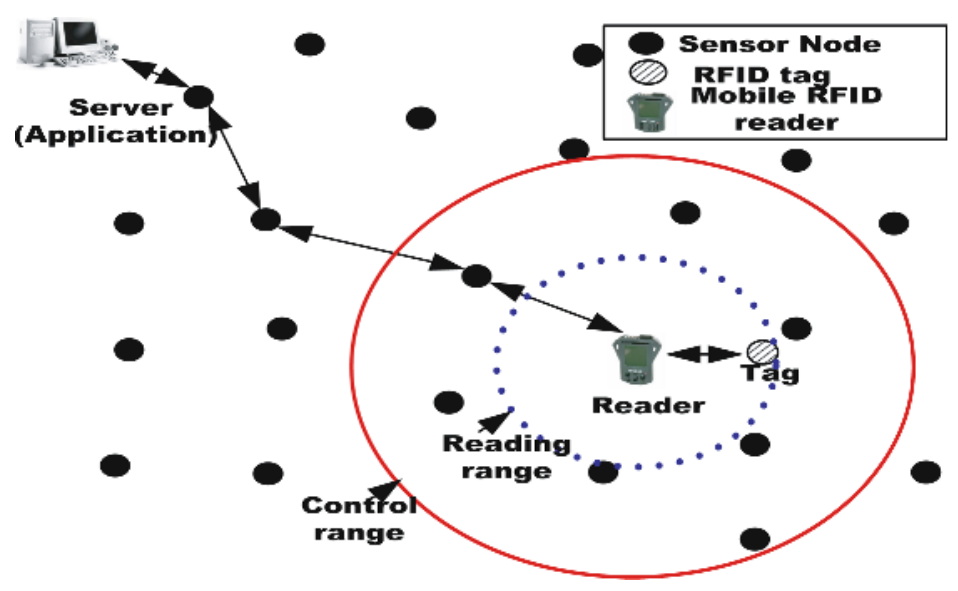

Fig. 2. RFID system architecture cooperated with sensor networks

The network model for the DiCa makes the following basic assumptions:

- While sensor nodes are stationary, readers are mobile.

- Each reader has two independent communication interfaces: the first is to aggregate information from tags and the second is to access to backbone 


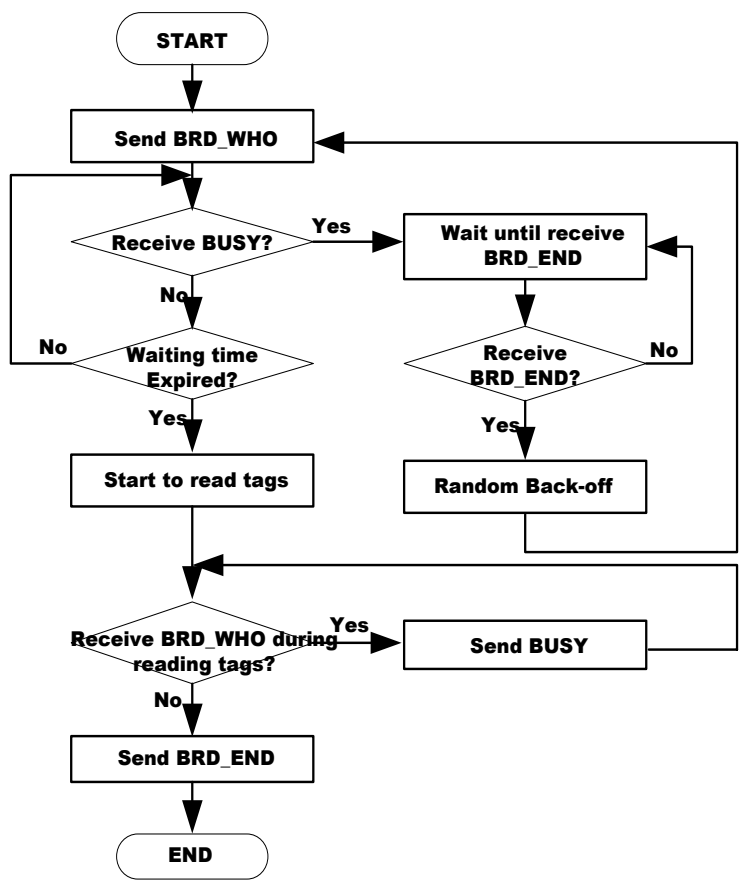

Fig. 3. Collision avoidance operation of DiCa

network or to communicate with other readers. In other words, the one is data channel and another is control channel.

- The sensor network is considered as an access networks(backbone network) as shown in fig. 2.

However, in this paper, the design or architecture of the routing protocol or data dissemination protocol for sensor networks is not considered because these topics are beyond the scope of this paper.

\subsection{Operation of DiCa}

The basic operation rule of DiCa is simple. Each reader contends with others through a control channel. The winner of the contention can read tags first through the data channel, and the other readers wait until the channel is idle. As soon as channel is idle, the other readers begin to read. In order to read tags without collision among readers, mobile readers have to contend repetitively with other readers. This task is achieved by exchanging three types of packets as follows.

Fig. 3 illustrates operation of DiCa in a reader system. A mobile reader, wanting to read tags, broadcasts the BRD_WHO message to neighbors to identify its presence of readers reading tags in the overlapped location. If another reader exists in the range, the reader will receive the BUSY message. This means the 


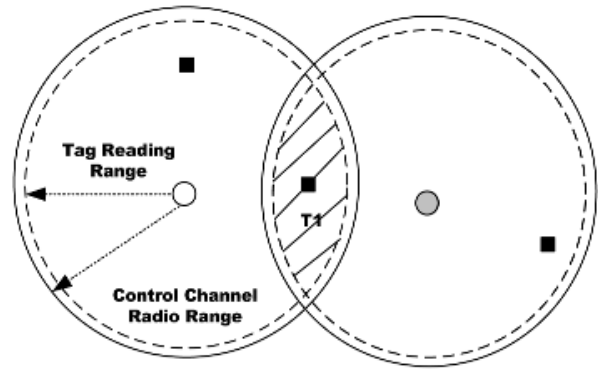

(a) HIdden Node Problem: Cholce of too small radlo range of control channel

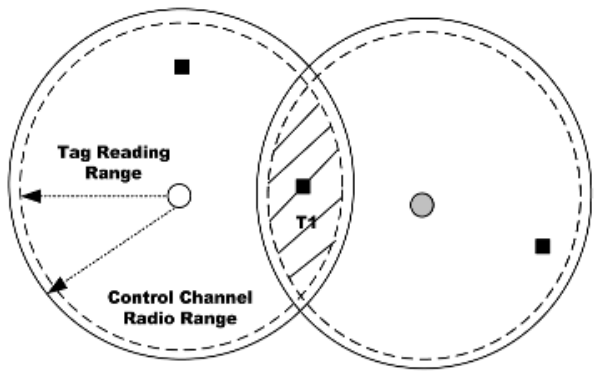

(a) HIdden Node Problem: Cholce of too small radlo range of control channel

Fig. 4. Examples of hidden and exposed node problem

data channel is already occupied by another reader. In this case, the reader should wait until reception of BRD_END, which means the data channel is free. However, if the reader does not receive a message over a certain period of time after transmitting BRD_WHO, the reader can begin to read, as the data channel is free.

\begin{tabular}{|l|l|}
\hline BRD_WHO & $\begin{array}{l}\text { Packet for identifying whether a reader reading tags exists in the } \\
\text { same network or not. }\end{array}$ \\
\hline BUSY & $\begin{array}{l}\text { Signal for answering to the reader that sends a BRD_WHO packet. } \\
\text { Busy means that a reader is now reading tags. }\end{array}$ \\
\hline BRD_END & $\begin{array}{l}\text { Packet for notifying that the channel is idle after completing read- } \\
\text { ing tags }\end{array}$ \\
\hline
\end{tabular}

\subsection{The Optimization of Communication Range}

DiCa uses distributed channel access using two independent communication channels. The distributed algorithm based on contention does not require centralized coordination or global synchronization. However, a contention-based algorithm, in particular, using two independent channels can lead to additional problems: the hidden and exposed node problems. Fig. 4 describes the hidden and exposed node problems. The hidden node problem is illustrated in fig. 4 (a). A collision occurs when the two readers attempt to concurrently read tags within a common set. The control messages cannot reach another reader because of a short control channel radio range.In addition, as presented in Fig. 4 (b), the exposed problem is caused by an excessive control channel radio range. Even though Reader R1 can read a tag without interference, R1 should be blocked until reading of $\mathrm{R} 2$ is completed.These two problems demonstrate that it is necessary that the communication range of the control channel is optimized, for minimizing hidden and exposed node problems. This means an optimized communication range can remove collisions from hidden nodes, and unnecessary delay from exposed nodes. 


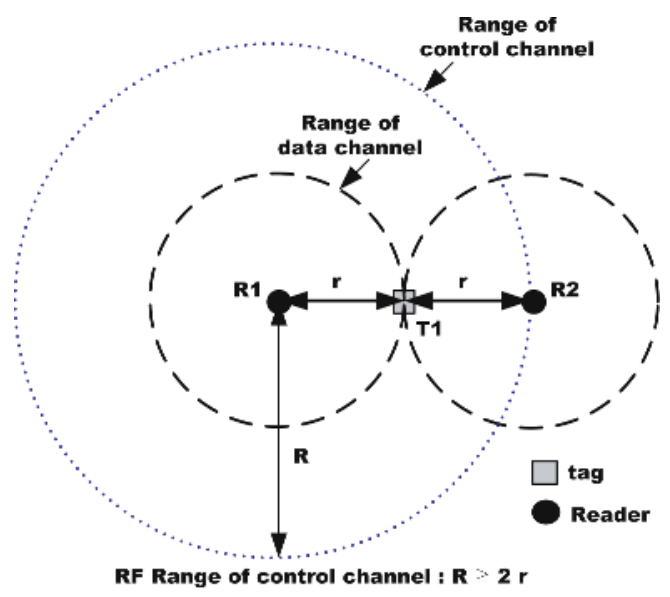

Fig. 5. The optimization of communication range in control channel

In order to avoid collision between readers, reader1 needs to identify all neighbors that can read the same tag. Therefore, $\mathrm{R}$, the communication range in the control channel, must be greater than 2 times $\mathrm{r}$, the communication range in data channel, as presented in Fig. 5. This means, when $\mathrm{R} 2 \mathrm{r}$, tag reading of reader 1 is reflected to $\mathrm{R} 2$ by control channel messages. Thus, the hidden node problem can be solved while the influence of the exposed problem is minimized. In addition, the DiCa considers the fact that each mobile reader can access to wireless backbone networks, specifically sensor networks. As described previously, RFID readers can communicate with the backbone through the control channel. It is important to note that the communication range required to access to the sensor network is considerably different from the range optimized for reader collision avoidance. However, the deployment pattern of sensor nodes is varied according to various environments. Therefore, DiCa supports variable power control of a control channel. This means optimal power intensity is applied only when the reading tag and general power intensity is used for communication with the sensor network.

\section{Performance Evaluations}

In this section, the proposed $\mathrm{DiCa}$ is evaluated in terms of the throughput and energy consumption. In addition to performance evaluation of the DiCa, comparative simulation is conducted. The DiCa is compared to 1-persistent CSMA, ALOHA, and Pulse, which are contention-based multiple access controls.

\subsection{Simulation Environments}

It is assumed that all readers in the network are capable of communicating with each other. In simulations, the following metrics, throughput (1) and dissipated energy (2), are observed. 


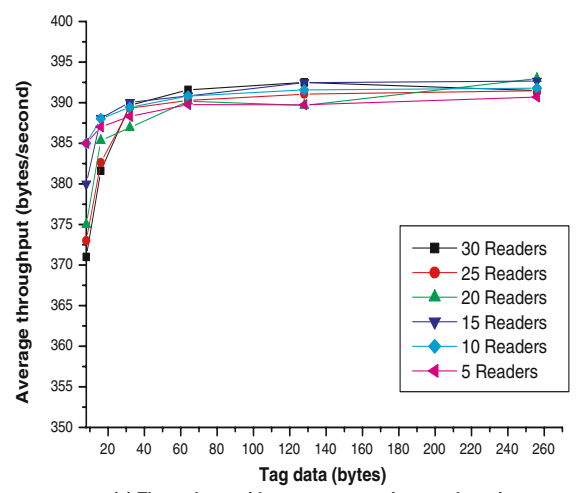

(a) Throughput with respect to varying tag data size

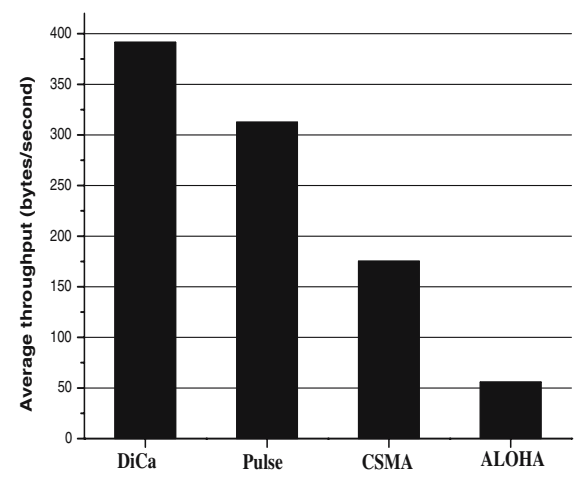

(b) Throughput comparison

Fig. 6. Throughput evaluations

$$
\text { Throughput }=\frac{\sum q u e r y \_s e n t \_s u c c e s s f u l l y\left(b y \_a l l \_r e a d e r s\right)}{\text { Total_time }}
$$

DissipatedEnergy $=\operatorname{activetime}(\mathrm{s}) \times \operatorname{current}(\mathrm{mA}) \times \operatorname{voltage}(\mathrm{V})$

\subsection{Simulation Results}

Through simulations, performance variations are observed in terms of throughput and total dissipated energy. In experiments, all readers in the network attempt to read tags concurrently every second and the number of readers is increased from 5 to 30 . Firstly, as presented in fig. 6 (a), the throughput, which means the number of successfully received data over all tag data during a unit time, is measured with respect to varying the tag data size $(8,16-128$ bytes). The result demonstrates that relatively low throughput at small-sized tag data (less than 32 bytes) gradually converges to a point, approximately 390 bytes/second, as tag data size increases. This is because DiCa requires sufficient time to exchange contention messages in the control channel. However, the smaller the data size, the shorter the reading time of the reader. Therefore, with a small data size, sufficient time to exchange contention messages may not be guaranteed, so the collision possibility between readers increases. However, if the tag data size is greater than 32 bytes, the control message exchange time is guaranteed and collision decreases without concern to the number of readers. In addition, it is noticeable that, compared with Pulse, CSMA, and ALOHA, the throughput of DiCa outperforms the other schemes, as demonstrated in fig. 6 (b). DiCa is originally intended to be used in mobile RFID readers. The power of a mobile RFID reader depends on its battery. Therefore, energy efficiency is one of the most important factors for wireless network consisting of mobile RFID readers. Fig. 7 (a) presents cumulative energy consumption with respect to time in each reader. Since DiCa avoids the collisions in a distributed manner among neighboring readers, the energy is proportionally increased with respect to the number 


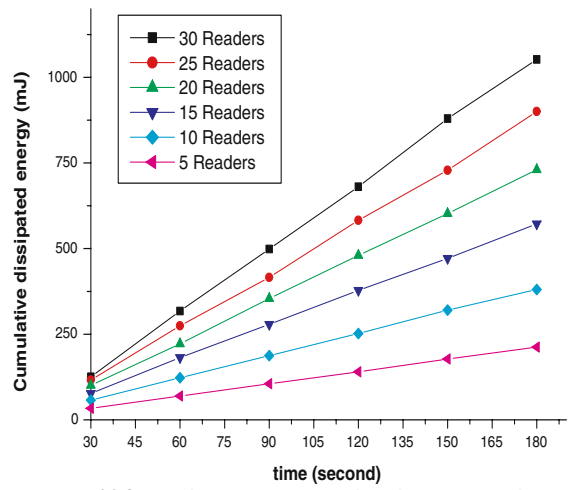

(a) Cumulative energy consumption with re-spect to time

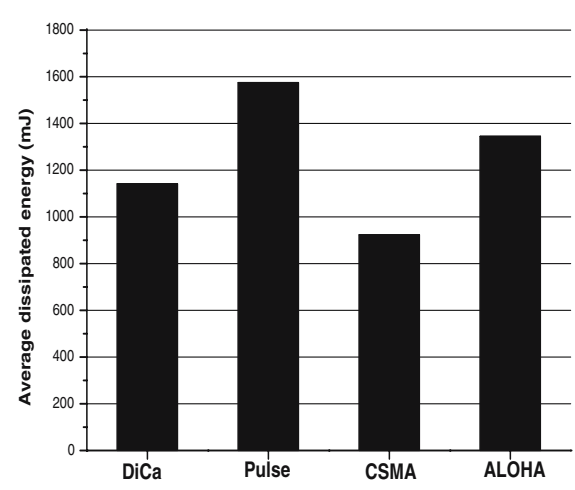

(b) Comparison of consumed energy

Fig. 7. Energy evaluations

of neighboring readers. Nevertheless, under the same condition, compared with other protocols, such as Pulse, CSMA, and ALOHA, DiCa demonstrates better energy efficiency. In particular, as presented in Fig. 7 (b), it appears that DiCa consumes greater energy than CSMA. However, considering only successful tag reading without collisions, DiCa is more energy-efficient since CSMA has a high failure rate because tag reading should be retried repetitively. These retries reveal unnecessary energy consumption in the network.

\section{Conclusion}

Concurrent access among multiple mobile readers results in reader collision, where a reader's transmission interferes with other readers. In this paper, a cooperative, distributed reader collision avoidance algorithm is introduced. The proposed DiCa is distinguished from prior studies in that DiCa does not require centralized coordination or global synchronization. In particular, DiCa is suitable for energy-efficient wireless mobile network environments since the proposed $\mathrm{DiCa}$ is not only capable of avoiding collisions, but also changing power state autonomously through simple distributed interactions between adjacent readers.

\section{References}

1. Sanjay E. Sarma, Stephen A. Weis, Daniel W. Engels, "RFID Systems and Security and Privacy Implications," Lecture Notes in Computer Science 2523, pp. 454-469, 2003.

2. Rolf Clauberg, "RFID and Sensor Networks," IBM white paper, 2004. http://www.m-lab.ch/rfid-workshop/ibm_paper.pdf.

3. Engels, D.W., Sarma, S.E., "The Reader Collision Problem," IEEE International Conference, 2002. 
4. Shailesh M.Birari, "Mitigating the Reader Collision Problem in RFID Networks with Mobile Readers," M.S. Dissertation in Indian Institute of Technology Bombay, 2005 .

5. Waldrop J., Engels D.W., Sarma S.E., "Colorwave: An Anti-collision Algorithm for the Reader Collision Problem," ICC'03, 2003.

6. Junius K. Ho, "Solving the reader collision problem with a hierarchical q-learning algorithm," M. S. Dissertation in Massachusetts Institute of Technology, February 2003. 